\title{
Hoppla - Digital Preservation Support for Small Institutions
}

\author{
Stephan Strodl, Florian Motlik, and Andreas Rauber \\ Vienna University of Technology, Vienna, Austria \\ \{strodl, motlik, rauber\}@ifs.tuwien.ac.at
}

\begin{abstract}
Digital information is of crucial value to a range of institutions, from memory institutions of all sizes, via industry and SME down to private home computers containing office documents, valuable memories, and family photographs. While professional memory institutions make dedicated expertise and resources available to care for their digital assets, SMEs and private users lack both the expertise as well as the means to perform digital preservation activities to keep their assets available and usable for the future.

This demo presents the Hoppla archiving system ${ }^{1}$ providing a digital preservation solution specifically for small institutions and small home/office settings. The system combines bit-stream preservation with logical preservation. It hides the technical complexity and outsource required knowledge and expertise in digital preservation.
\end{abstract}

\section{Hoppla Concepts}

Hoppla $^{2}$ - (Home and Office Painless Persistent Long-term Archiving) presents a new approach to automated digital preservation systems that are suited to the needs of small institutions. It considers the abovementioned issues and tackles the emerging challenges to ensure the accessibility and availability of digital objects in the future. The conceptual design of the archiving system was presented in [1].

The underlying principle of the system is providing a best effort solution with respect to the available technology and skills of the users. With Hoppla we are currently developing a solution that combines back-up and fully automated migration services for data collections in small institution settings. The logical preservation obtains practical preservation solutions based on expert advise via an automated web-service-based update of preservation plans. This allows outsourcing of required digital preservation expertise for the institutions.

The modular architecture of the Hoppla system is influenced by the OAIS reference model(ISO 14721:2003). As shown in Figure 1, the six core modules of the system are: acquisition, ingest, data management, preservation, access and storage.

\footnotetext{
${ }^{1}$ Part of this work was supported by the European Union in the 6th framework Program, IST, through the PLANETS project, contract 033789.

2 http://www.ifs.tuwien.ac.at/dp/hoppla
} 


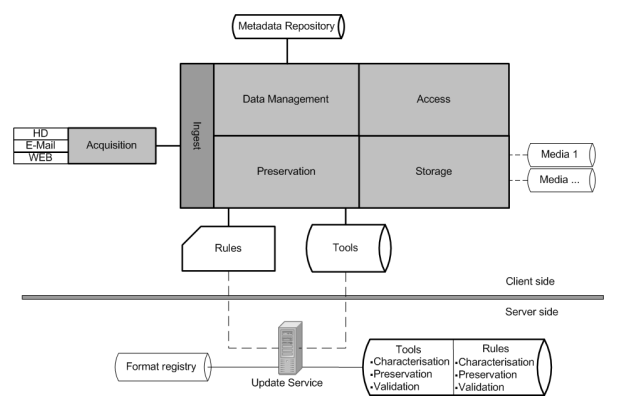

Fig. 1. Architecture of the Hoppla System

The application flow of Hoppla starts with the ingest of new objects from source media, the object's formats are identified and a collection profile is created. Hoppla supports the acquisition of data from different sources via an API for acquisition plugins. The use of plugins allows to support all kinds of storage media and current as well as future data sources.

Based on the collection profile suitable preservation rules are recommended by the web update service. For privacy reasons, the user can define the level of detail of the collection profile that is provided to the web update service. This way the user has strict control, which and how much metadata is sent to the server. New preservation rules and services are downloaded from the web service and preservation actions are performed on the client side. Depending on the configuration all migration steps can run fully automated and transparent for the user, or allow individual interaction and fine-tuning.

The new objects including the resulting objects from preservation actions are ingested into the collection and stored on storage systems. A plugin infrastructure is used to support different storage media, e.g. online and off-line media in both write-once as well as rewritable forms.

By not just using bit storage, but also migration with corresponding web update services HOPPLA can help SOHO institutions and private users to have safe backups of all their data. Not just the physical layer of a file is preserved, but also its logical layer. The fully modular development of the Hoppla software provides facilities to further extend specific modules of the system or integrate other existing system (e.g. storage systems) into the Hoppla system.

\section{References}

1. Strodl, S., Motlik, F., Stadler, K., Rauber, A.: Personal \& SOHO archiving. In: Proc. of the 8th JCDL 2008, Pittsburgh PA, USA. ACM, New York (2008) 